\title{
Outcome of Anterior Cruciate Ligament Reconstruction Surgery in Tuanku Fauziah Hospital, Kangar, Malaysia
}

\author{
Logheswaren Suppiah, Ewe Juan Yeap, Mohamad Yazid Bin Din
}

\section{ABSTRACT}

Introduction: The anterior cruciate ligament $(A C L)$ is the primary stabilizer of the knee against anterior transposition. Injuries are commonly seen in the young and active and are associated with considerable reduction in quality of life, and its numbers are increasing.

Purpose: To evaluate the outcome of $A C L$ reconstructions done in Tuanku Fauziah Hospital, Malaysia.

Materials and methods: It is a single center retrospective study. Thirteen patients who underwent ACL reconstructions were interviewed and scored based on Lysholm and Tegner activity scale. These patients completed are habilitation protocol regime which comprised of basic rehabilitation modalities. They were assessed by personally and by telephone. Average postoperative duration was 34 months.

Results: Mean Tegner scores were 6.1 and Lysholm scores were 85.8. Most patients were satisfied with their current level of activity. These results are almost at par with studies done in highly specialized centers despite having only basic rehabilitation facilities.

Conclusion: Good outcomes are obtainable in basic general hospitals and not only in subspecialized centers.

Keywords: ACL reconstruction, Outcomes, Lysholm score, Tegner activity scale.

How to cite this article: Suppiah L, Yeap EJ, Bin Din MY. Outcome of Anterior Cruciate Ligament Reconstruction Surgery in Tuanku Fauziah Hospital, Kangar, Malaysia. J Postgrad Med Edu Res 2013;47(2):103-106.

Source of support: Nil

Conflict of interest: None declared

\section{INTRODUCTION}

The anterior cruciate ligament (ACL) functions primarily to prevent anterior translation and rotation of the knee. ACL injuries are most often seen in the young and active population. Most ACL injuries are sustained during sports activities. Rotation of the femur and tibia in opposite directions under full body weight leads to the ACL being injured. This mechanism is usually seen when there is sudden turning to change direction while running, otherwise known as a cutting maneuver. Common complaints usually range from pain, swelling, instability and in severe cases, loss of function.

Data pertaining to the burden of disease are scarce. Registry data from the Scandinavian countries revealed the total number of primary ACL reconstructions performed in
Sweden, Norway and Denmark was 7,311, 5,329 and 4,972 respectively between 2004 and 2007 for a population of 9.1, 4.7 and 5.4 million. ${ }^{1}$ Our population is currently estimated to be 28 million. This should translate to quite a large number of ACL injuries requiring reconstruction.

Reconstruction of the injured ACL is rarely undertaken in primary centers due to various reasons, such as lack of experience and poor rehabilitative facilities. This probably results in a backlog of cases and long waiting lists resulting in patients having to refrain from their recreational activities. The scenario is even worse for competitive athletes who stand to lose their source of income when they are not able to perform optimally. Unfortunately we do not have any local data for comparison.

At the moment, there are only a handful of public hospitals in Malaysia where there are fellowship trained sports surgeons who perform these reconstruction surgeries. There are probably more fellowship trained sports surgeons in the private sector. Hence, the burden of disease falls on the government sector. Patients have to wait for months before they are scheduled for surgery and along the way many just adapt to their disability and end up not having the surgery at all. So these surgeries are being increasingly performed in hospitals throughout the country by surgeons who are not formally fellowship trained. These surgeons either undergo other forms of training, such as short attachments with fellowship trained surgeons, operating under supervision of trained surgeons and cadaveric workshops. There is a large industry driven practice of offering short training stints. Every year during the National Orthopedic Association's Scientific Meetings, there are usually saw-bone workshops.

The main purpose of this study is to assess the outcome of the ACL reconstruction surgeries undertaken in Tuanku Fauziah Hospital, Kangar, Malaysia. This is the only hospital in the state of Perlis, but there is no subspecialty in sports surgery and the physiotherapy team provides only basic rehabilitative services. Our secondary aim is to audit our cases and to see if there is a need to make any improvements.

\section{MATERIALS AND METHODS}

This is a retrospective study whereby we traced the surgical log of the corresponding author, who performed the ACL 
reconstructions. There were a total of 23 patients who underwent ACL reconstruction surgery from July 2005 to January 2010. We included both single and double bundle reconstruction surgeries. Out of the 23 patients, we only managed to trace outpatient cards and case records of only 13 patients. The remaining 10 patients were excluded due to incomplete records as the hospital was flooded thrice since 2005.

Majority of the patients participated in regular recreational sporting activities, such as jogging, futsal and badminton. There was one patient who represented the state in 'sepak takraw' and happens to be the sole female participant in this study. All patients had symptomatic instability, more pronounced during sports, and were not able to participate in regular sports activities. On examination, all patients had an anterior drawer laxity of grade II and above. Not all patients had an MRI preoperatively, but those that had were reported as having complete tears.

All patients underwent reconstruction surgery with a bioabsorbable screw and endobutton system (Smith and Nephew ${ }^{\circledR}$ and Arthrex ${ }^{\circledR}$ ). Hamstring tendon grafts were used for all the study subjects. Eight patients underwent single bundle reconstruction whereas five had double bundle reconstruction.

We called up the 13 patients and all of them agreed to participate in the study but only four patients were able to come to the clinic to be examined. IKDC scoring, Lysholm scoring and Tegner activity scale scoring were done for them. The remaining patients were interviewed by telephone and were scored using the Lysholm score and Tegner activity scale. The IKDC scores were not included in this study since we were not able to score the majority of the cases.

Intraoperatively, all patients underwent examination under anesthesia to confirm the diagnosis of ACL tears. The reconstruction surgeries were carried out arthroscopically. Semitendinosus and gracilis tendon grafts were harvested and whip stitch sutured at both ends. The grafts were then folded in half and tensioned for 20 minutes. Meniscal integrity was evaluated intraoperatively, and it was either repaired or debrided as deemed necessary. The joint was also explored to look for other pathologies. The tibial and femoral tunnels were drilled via the standard technique. The grafts were secured to the femoral end with an endobutton and the tibial end was secured with a bioabsorbable screw.

Postoperatively, patients were put on a knee brace and crutches. They had to complete a physiotherapy regime based on a standard ACL reconstruction rehabilitation protocol.
The patients were scored at an average of 33.6 months since after they had their reconstruction surgery. The time between surgery and evaluation ranged from 20 to 56 months. We evaluated the patients with the Lysholm and Tegner activity scores as it had proven validity as patient administered scores at early time points after treatment of ACL injuries. ${ }^{2}$

\section{RESULTS}

There were 12 male patients and only one female patient in this group. The age of the patients who underwent ACL reconstruction surgery ranged from 16 to 38 years old with a mean of 23.9 years (standard deviation: 8.0). There was a wide range in the duration from injury to surgery with the earliest being 4 months postinjury and the longest was 55 months after initial injury. The mean duration was 21.5 months (standard deviation: 14.7). Seven patients underwent surgery on the right knee whereas the remaining six had surgery done on the left knee.

A total of $62 \%$ of the patients (eight patients) sustained the injury during a game of football. The remaining patients sustained the injury during badminton, hockey, rugby, 'sepak takraw' and taekwondo. There was one patient who sustained the injury due to a motor vehicle accident.

Five patients (38\%) had undergone double bundle reconstruction; eight patients (62\%) had single bundle reconstruction.

Intraoperatively, six patients had lateral meniscus tears and four had medial meniscus tears. One patient had tears on both menisci. Meniscal tears were debrided and repaired. The remaining two patients had intact menisci but had synovitis. Four patients had osteochondral defects which were treated with bone marrow stimulation techniques such as microfracture.

The Lysholm score for the patients ranged from 76 to 90 with a mean of 85.8. As for the Tegner activity scale, patients had a mean preinjury score of 7.6 and a postoperative mean score of 6.2. During the interview, most of the patients had no limp or locking symptoms. They did not require any form of support for ambulation. Pain, swelling and instability were only experienced during severe exertion. Some also had slight problems when walking upstairs and squatting.

Based on the Tegner activity scale, most patients were involved in recreational activities (Tegner activity scale 7) but we noticed postoperatively that they had reduced their level of activity to activity scale 4 . Fear of reinjury was cited as the commonest cause for this transition. However, there was one patient who was a state representative in 'sepak takraw' and at the time of this study; she was back to active competition. 
There were no postoperative surgical complications. There were no reruptures and no patient presented for any new procedures.

A summary of the parameters which were evaluated in this study is shown in Table 1.

\section{DISCUSSION}

In accordance with the main aim of this study which is to highlight the outcome of ACL reconstruction surgery in a nonsports specialized center, we compared the results of this study with other similar studies which had used the same outcome parameters, the Lysholm score and the Tegner activity scale.

Ejerhad et $\mathrm{al}^{3}$ reported a preoperative Lysholm score of 68 and a postoperative score of 90 with reconstruction surgery done using hamstring grafts. Engelbertsen et $\mathrm{al}^{4}$ which emphasized on primary ACL reconstructions also reported a preoperative Lysholm score of 74 and at 2 years follow-up, a score of 73 was obtained (Fig. 1). Gobbi et $\mathrm{al}^{5}$ had a preoperative Tegner activity scale of 7.5, and postoperative score of 6.0 for ACL reconstructions using hamstring grafts (Fig. 2).

Most of the studies that we compared to adhered to a standard rehabilitation regime which involved putting the patient on a knee brace and allowing gradual flexion of the

\begin{tabular}{lccc}
\multicolumn{4}{c}{ Table 1: Overview of parameters } \\
\hline Parameters & Range & Mean & Standard deviation \\
\hline $\begin{array}{l}\text { Age (years) } \\
\text { Injury to surgery } \\
\text { duration (months) }\end{array}$ & $14-38$ & 23.9 & 8.0 \\
$\begin{array}{l}\text { Surgery to evaluation } \\
\text { duration (months) }\end{array}$ & $22-54$ & 21.5 & 14.7 \\
$\begin{array}{l}\text { Tegner score } \\
\text { (postoperative) }\end{array}$ & $4-10$ & 33.8 & 11.6 \\
Lysholm score & $76-95$ & 85.8 & 1.9 \\
\hline
\end{tabular}

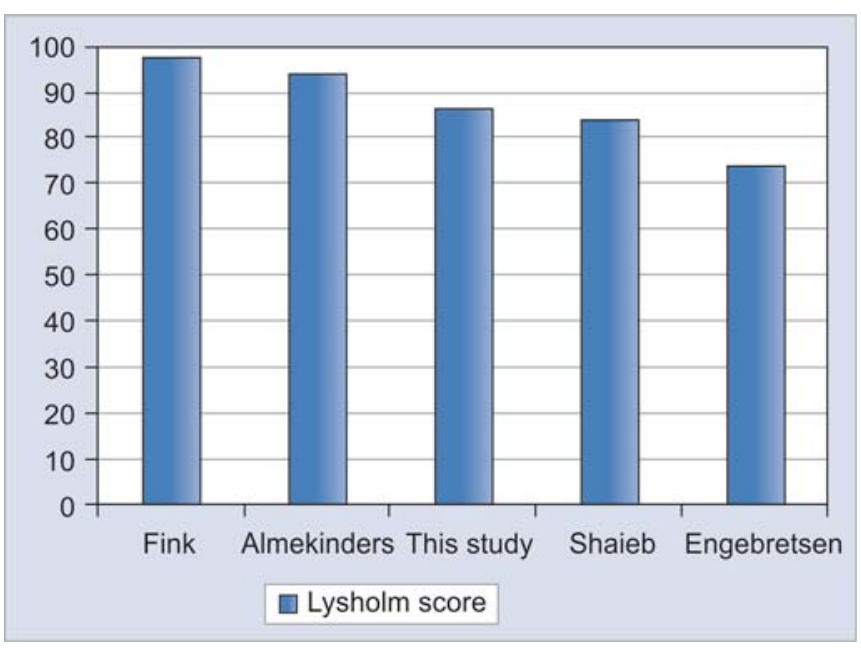

Fig. 1: Comparison of lysholm scores affected knee joint. Fink et $\mathrm{al}^{6}$ had used a continuous passive motion machine for postoperative rehabilitation.

The physiotherapy department in our hospital adopts an ACL rehabilitation protocol in which they see the patients at least twice a week for the first 2 weeks followed by weekly visits. Athletes who have access to their own rehabilitation facilities are only seen once a week for the first 2 weeks and then fortnightly. Modes of rehabilitation here consist of the treadmill and stationary bicycle. Weights are used for strengthening. This regime does not differ much from the other centers that we compared to except that we do not have a continuous passive motion device. Our physiotherapy team is also multidisciplinary, taking care of stroke patients, inpatients and general orthopedic patients. There is no dedicated personnel for just ACL rehabilitation as compared to other specialized centers which have specific open and closed chain exercises for postoperative strengthening and proprioception. $^{7}$

Being the only hospital in the state, we get patients who are professional athletes who have access to their own physiotherapy services. Collaboration between the team physiotherapy and hospital physiotherapy department is something that could be looked into.

The results that we achieved are at par with some of the international studies that were conducted as seen in Figures 1 and 2. We were able to obtain a mean Tegner score of 85.8 as compared to 85 by Shaieb et al ${ }^{8}$ and 94 by Almekinders et al. ${ }^{9}$ We had better scores when compared to Engebretsen et al in 1990. ${ }^{4}$ This could be an indicator that the quality and standards of practice have improved over the past 2 decades. We also managed a slightly better Lysholm score as compared to Gobbi et al. ${ }^{5}$

Current orthopedic surgeons are trained in arthroscopy as part of the core procedures. With the increase in the burden of disease, it is a step up to perform an ACL

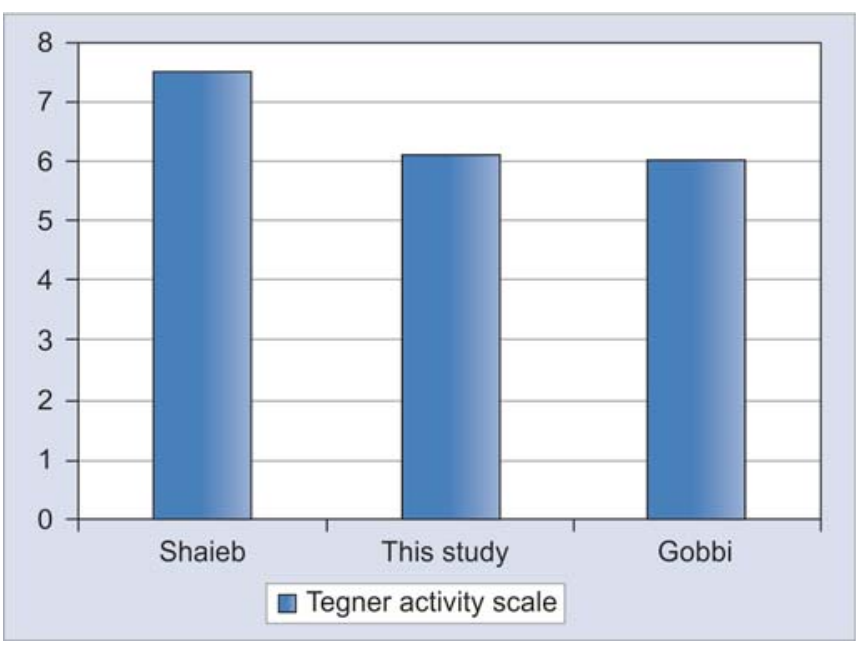

Fig. 2: Comparison of Tegner activity scale scores 
reconstruction. Limitations of this study include its retrospective design, the small number of cases with nearly half being excluded and no comparison group.

\section{CONCLUSION}

We were able to achieve results comparable with that of international studies conducted in centers with specialized expertize and rehabilitation services. This proves that ACL reconstructions done in nonsports specialized centers can yield results which are at par with highly specialized centers.

\section{ACKNOWLEDGMENT}

We would like thank our Director General of Health, for permitting us to conduct and publish this study.

\section{REFERENCES}

1. Granan LP, Forssblad M, Lind M, Engebretsen L. The Scandinavian ACL registries 2004-2007: Baseline epidemiology. Acta Orthop 2009 Oct 1;80(5):563-67.

2. Briggs KK, Lysholm J, Tegner Y, Rodkey WG, Kocher MS, Steadman JR. The reliability, validity, and responsiveness of the Lysholm score and Tegner activity scale for anterior cruciate ligament injuries of the knee: 25 years later. Am J Sports Med 2009 May;37(5):890-97.

3. Ejerhed L, Kartus J, Sernert N, Köhler K, Karlsson J. Patellar tendon or semitendinosus tendon autografts for anterior cruciate ligament reconstruction? A prospective randomized study with a 2-year follow-up. Am J Sports Med 2003 Jan-Feb; 31(1):19-25.

4. Engebretsen L, Benum P, Fasting O, Mølster A, Strand T. A prospective, randomized study of three surgical techniques for treatment of acute ruptures of the anterior cruciate ligament. Am J Sports Med 1990 Nov-Dec;18(6):585-90.

5. Gobbi A, Diara A, Mahajan S, Zanazzo M, Tuy B. Patellar tendon anterior cruciate ligament reconstruction with conical press-fit femoral fixation: 5-year results in athletes population. Knee Surg Sports Traumatol Arthrosc 2002 Mar;10(2):73-79.

6. Fink C, Benedetto KP, Hackl W, Hoser C, Freund MC, Rieger M. Bioabsorbable polyglyconate interference screw fixation in anterior cruciate ligament reconstruction: A prospective computed tomography-controlled study. Arthroscopy 2000 Jul-Aug;16(5):491-98.

7. Tow BP, Chang PC, Mitra AK, Tay BK, Wong MC. Comparing 2-year outcomes of anterior cruciate ligament reconstruction using either patella tendon or semitendinosus-tendon autografts: A non-randomised prospective study. J Orthop Surg (Hong Kong) 2005 Aug;13(2):139-46.

8. Shaieb MD, Kan DM, Chang SK, Marumoto JM, Richardson AB. A Prospective randomized comparison of patellar tendon versus semitendinosus and gracilis tendon autografts for anterior cruciate ligament reconstruction. Am J Sports Med 2002 MarApr;30(2):214-20.

9. Almekinders LC, de Castro D. Fixed tibial subluxation after successful anterior cruciate ligament reconstruction. Am J Sports Med 2001 May-Jun;29(3):280-83.

\section{ABOUT THE AUTHORS}

\section{Logheswaren Suppiah}

Medical Officer, Department of Orthopedics, Tuanku Fauziah Hospital, Kangar, Perlis, Malaysia

\section{Ewe Juan Yeap (Corresponding Author)}

Consultant, Department of Orthopedics and Traumatology Tuanku Fauziah Hospital, Clinical Research Centre Jalan Kolam Kangar-01000, Perlis, Malaysia, Phone: 019-3533108, e-mail: ejyeap@yahoo.com

\section{Mohamad Yazid Bin Din}

Consultant, Department of Orthopedics, Tuanku Fauziah Hospital Kangar, Perlis, Malaysia 